\title{
Cooling a Thermal Cloud to a Supersolid
}

\author{
When a cloud of cold atoms goes through a supersolid phase transition, its \\ coexisting superfluid and crystalline phases do not emerge \\ simultaneously. \\ By Chinmayee Mishra
}

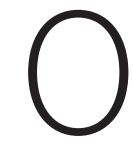
bserving a supersolid-a state of matter that has both superfluid and solid properties-has been a challenge for decades. The constituent particles of a supersolid form a rigid, ordered structure but can also flow without dissipating energy. This paradoxical behavior was observed in cold-atom experiments only in the last five years [1-5].

Researchers typically make cold-atom supersolids starting from superfluid phases, leaving open the question of how supersolidity appears from other phases. Now, a team led by Francesca Ferlaino at the University of Innsbruck, Austria, has shown that when starting from a thermal gas, a supersolid's crystalline and superfluid orders arrive and decay sequentually

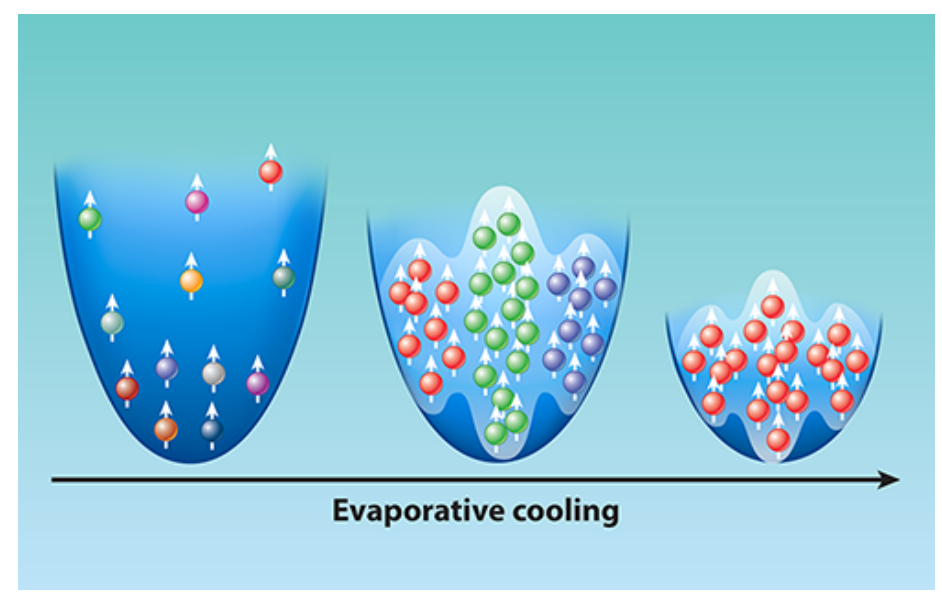

Figure 1: (Left to right) The solid and superfluid properties of a cold-atom supersolid emerge consecutively when a cold-atom thermal gas condenses into this phase.

Credit: APS/Carin Cain at the birth and death of a supersolid [6]. The results suggest that temperature plays a definitive role in the appearance of supersolidity.

The existence of a supersolid state was first proposed in 1957, long before experimentalists knew how to achieve it [7]. The first experiments focused on realizing supersolidity in helium, with researchers trying to move a helium sample from a solid, crystalline state to a supersolid one. Lacking unambiguous success, researchers started looking for alternative systems to obtain a supersolid [8].

An ensemble of cold atoms is one alternative system. Cold atoms offer an ideal environment in which to create supersolids, as researchers can engineer their atom-atom interactions in a controlled manner. Cold-atom physcists have shown that they can manipulate interactions to generate a supersolid starting from a cold-atom setup, specifically a dipolar Bose-Einstein condensate (BEC). The properties of a dipolar BEC are governed by short-range repulsive interactions and by long-range dipole-dipole interactions between atoms. Typically, the supersolid phase is achieved by quenching the magnitude of the short-range interaction, which induces a density modulation on top of the system's already existing superfluid phase.

Using this method, supersolid phases have been achieved in cold-atoms that were intially superfluids-the density modulation added the solid behavior $[1,3,5]$. This route to making a supersolid, however, has a number of issues that restrict the study of finite temperature effects. Ferlaino and her group now show that they can study the effect of temperature 
on the density modulation by obtaining a cold-atom supersolid directly from a thermal gas [6].

In their experiments, the team used a technique called evaporative cooling. They trapped a gas of roughly $10^{5}$ dysprosium atoms using lasers. The lasers created a cigar-shaped optical barrier over which the atoms could escape if their energy was high enough. As atoms escaped, the temperature of the remaining atoms decreased to several hundred nanokelvin. The team then lowered the barrier height (they did this at different rates in different experiments), reducing the system's temperature further until it was low enough that the atoms condensed into a supersolid. To study the system, the team used two imaging techniques: Faraday phase-contrast imaging, which captured density modulations in the system, and time-of-flight (TOF) imaging, which provided information about the global phase coherence.

By lowering the trap height quickly (in this case in just $225 \mathrm{~ms}$ ) and then allowing the resulting atom cloud to evolve, the team was able to probe how the supersolid formed without any influence from the changing trap height. Under this condition, they found that the density modulations that formed looked like droplets-indicative of a supersolid. These modulations appeared on a timescale of approximately $150 \mathrm{~ms}$. This timescale corresponds to the equilibration time of the system and is set by the rate of evaporation, which in turn depends on the elastic collision rate between atoms.

By lowering the trap height slowly (in a time interval of $500 \mathrm{~ms}$ ), the team was able to study whether the translational symmetry or the phase symmetry broke first: Did the gas first turn into a solid or a superfluid? To answer that question they used the "phasor function" extracted from the TOF measurements at different time intervals $[5,9]$. The magnitude of the phasor carries information about the density modulation, while its phase gives information about coherence.

Using this information, they discovered that the density modulations that correspond to the solid phase built up first. Approximately 40 ms later, the superfluid behavior sets in, which they saw in the devlopment of global phase coherence. The 40-ms delay in the onset of superfludity can be attributed to the existence of finite temperature effects that inhibit global phase coherence of the system.
The arrival of the superfluid behavior marked the atoms' entry into the supersolid phase, which lasted longer than $1 \mathrm{~s}$. After this time, atom losses from the trap caused the supersolid to slowly disappear. During the system's transition out of the supersolid, continuous translational symmetry was restored first, as seen by the vanishing of the density modulations. The broken phase symmetry associated with superfluidity persisted for a much longer time, signifying that even in the supersolid's death, the two symmetries were not restored simultaneously.

The results of Ferlaino and her colleagues help to solidify the notion that our understanding of supersolids is still in its infancy. As yet, no supersolid phase diagram exists with temperature as a parameter, and it is unknown how quantitative changes in temperature link in with density modulation, the breaking of continuous translational symmetry in the system, and global phase coherence. Previous works have alluded to the key role that temperature plays in the formation of the density modulations needed to create a supersolid [4]. By successfully achieving a supersolid state from a thermal cloud, the new work adds weight to that conclusion, and it will be interesting to see what theoretical models predict on this point. A detailed understanding of density and phase excitations close to this transition will deepen our knowledge about supersolids. Undeniably, exciting times are ahead for researchers working in dipolar BECs and supersolids.

Chinmayee Mishra: Indian Institute of Technology, Gandhinagar, India

\section{REFERENCES}

1. J.-R. Li et al., "A stripe phase with supersolid properties in spin-orbit-coupled Bose-Einstein condensates," Nature 543, 91 (2017).

2. J. Léonard et al., "Supersolid formation in a quantum gas breaking a continuous translational symmetry," Nature 543, 87 (2017).

3. L. Tanzi et al., "Observation of a dipolar quantum gas with metastable supersolid properties," Phys. Rev. Lett. 122, 130405 (2019).

4. F. Böttcher et al., "Transient supersolid properties in an array of dipolar quantum droplets," Phys. Rev. X 9, 011051 (2019).

5. L. Chomaz et al., "Long-lived and transient supersolid behaviors in dipolar quantum gases," Phys. Rev. X 9, 021012 (2019).

6. M. Sohmen et al., "Birth, life, and death of a dipolar supersolid," 
Phys. Rev. Lett. 126, 233401 (2021).

7. E. P. Gross, "Unified theory of interacting bosons," Phys. Rev. 106, 161 (1957).

8. M. Boninsegni and N. V. Prokof'ev, “Colloquium: Supersolids:
What and where are they?" Rev. Mod. Phys. 84, 759 (2012).

9. P. Ilzhöfer et al., "Phase coherence in out-of-equilibrium supersolid states of ultracold dipolar atoms," Nat. Phys. 17, 356 (2021). 